\title{
Mixing and moving in the gut
}

Stevens RJ, Publicover NG, Smith TK. Induction and organization of $\mathrm{Ca}^{2+}$ waves by enteric neural reflexes. Nature 1999;399:62-6.

\section{Abstract}

The motility of the gastrointestinal tract consists of local, non-propulsive mixing (pendular or segmental) and propulsive (peristaltic) movements. It is generally considered that mixing movements are produced by intrinsic pacemakers which generate rhythmic contractions, and peristalsis by intrinsic excitatory and inhibitory neural reflex pathways, but the relationship between mixing and peristalsis is poorly understood. Peristalsis is compromised in mice lacking interstitial cells of Cajal, suggesting that these pacemaker cells may also be involved in neural reflexes. Here we show that mixing movements within longitudinal muscle result from spontaneously generated waves of elevated internal calcium concentration which originate from discrete locations (pacing sites), spread with anisotropic conduction velocities in all directions, and terminate by colliding with each other or with adjacent neurally suppressed regions. Excitatory neural reflexes control the spread of excitability by inducing new pacing sites and enhancing the overall frequency of pacing, whereas inhibitory reflexes suppress the ability of calcium waves to propagate. We provide evidence that the enteric nervous system organizes mixing movements to generate peristalsis, linking the neural regulation of pacemakers to both types of gut motility.

\section{Comment}

The mammalian intestinal tract when isolated in vitro remains a complex integrated system of musculature, secretory epithelium, blood vasculature, and enteric nervous system that may be influenced by release of mediators from enteroendocrine cells and elements of the enteric immune/inflammatory system. When isolated in vitro the small and large intestine is therefore amenable to study at multiple levels of biological organisation. Stevens et al investigated fluorometric changes in $\mathrm{Ca}^{2+}$ in a window $(\sim 1$ $\mathrm{mm}^{2}$ ) of longitudinal muscle, myenteric plexus and presumed network of interstitial cells of Cajal exposed by dissection in a $4 \mathrm{~cm}$-long flat sheet preparation of guinea pig distal colon and extrapolated their observations to mixing and propulsive motility in the whole gut.

The results confirm the critical importance of $\mathrm{Ca}^{2+}$ in excitation-contraction coupling in smooth muscle and as a charge carrier in the generation of action potentials in both the muscle and myenteric neurones. Calcium waves, as mentioned in the title, are presumed to be a reflection of the propagation of muscle action potentials and associated muscle contraction. The authors' descriptions of the spread of the $\mathrm{Ca}^{2+}$ waves in the $1 \mathrm{~mm}^{2}$ patch of tissue and collisions of waves as they spread into the same region from opposite directions show in another format the well known functional syncytial properties of intestinal smooth muscle and cell-to-cell electrical conduction across gap junctions in this tissue.

The authors' conclusions, drawn for whole organ motility (i.e., mixing and peristaltic propulsion) from observations of $\mathrm{Ca}^{2+}$ dynamics in a $1 \mathrm{~mm}^{2}$ patch of longitudinal muscle with adherent myenteric plexus, are rather speculative. Distal large intestinal motility in the guinea pig compacts liquid faeces into solid pellets and changes pattern to propel the pellets during defecation. How the reported behaviour of $\mathrm{Ca}^{2+}$ relates to these functions is elusive. The use of undefined terms such as "pacing-site density" and "asymmetric shearing forces" is not helpful. The conclusion that the longitudinal and circular intestinal muscle coats always contract synchronously during enteric neural reflexes is inconsistent with the facts of physical geometry of tubular viscera and current concepts of neural control of propulsive motility.

Kottegoda, in $1969,{ }^{1}$ and Gregory and Bentley, in $1968,{ }^{2}$ showed that changes in the longitudinal and circular dimensions of tubular preparations of intestine in vitro occurred $180^{\circ}$ out of phase. Gregory and Bentley concluded that lengthening of the longitudinal axis was a passive mechanical consequence of circular muscle contraction, whereas Kottegoda interpreted his findings as a reflection of reciprocal inhibitory and excitatory neural reflex control of the two muscle coats. Wood and Perkins, in $1970,{ }^{3}$ supported the view of Gregory and Bentley by demonstrating that tubular segments of intestine behave physically as a cylinder with constant surface area (i.e., surface area $\left.=\pi r^{2} \times 1\right)$. The geometry of the system predicts that a decrease in radius will be accompanied by an increase in length and vice versa. Potassium chloride $(\mathrm{KCl})$ induced contractions of the circular muscle coat to small predetermined radii were shown to evoke passive increases in length of the intestinal segment by as much as $180 \% .^{3}$ In intact intestinal segments in $\mathrm{KCl}$, both muscle coats contract synchronously; however, the greater force generated by the circular muscle (e.g., the wet weight of cat small intestinal circular muscle is $3-5$ times that of the longitudinal) shortens the radius and forces elongation of the longitudinal axis in the face of a contracting longitudinal muscle coat. In effect, the anatomical arrangement of the circular and longitudinal muscles coats and the laws of geometry dictate that they are antagonistic muscles (i.e., shortening of one opposes shortening of the other). Even flat-sheet intestinal preparations appear to conform to these laws with shortening of one axis accompanied by obligatory elongation of the other. ${ }^{4}$

The authors' treatment of the peristaltic reflex fits the classic description of contraction of the intestinal circular muscle coat orad and inhibition of the circular muscle coat caudad to the point of stimulation. Behavior of the longitudinal muscle during the reflex is incompletely tackled in their paper. Current concepts view the basic reflex circuit as invariably forming propulsive and receiving 
segments irrespective of whether propulsion is in the orthoor retrograde direction. ${ }^{67}$ Irrespective of the direction of propulsion, the receiving segment always leads and converts to a propulsive segment as the next segment becomes a receiving segment during propagation of the propulsive complex. Excitatory motor neuronal input shortens the longitudinal muscle in the receiving segment while inhibitory motor input relaxes the circular muscle. The tubular geometry and anatomical arrangement of the two muscle coats ensures an expansion of the lumen (i.e., increase in radius) of the receiving segment to accommodate the advancing volume of luminal contents. Kosterlitz ${ }^{8}$ referred to the shortening of the longitudinal muscle in the receiving segment as the preparatory phase of the peristaltic reflex. Videomatic observations of faecal pellet propulsion in the isolated rodent large intestine show the contraction of the longitudinal muscle and shortening of the receiving segment ahead of the advancing pellet ${ }^{9}$ and observations of peristaltic propulsion in the oesophagus show shortening of the receiving segment as well. ${ }^{10}$

Behaviour of the longitudinal muscle coat in the propulsive segment is less clear. There is no question that the segment elongates as the circular muscle contracts; the question is whether the longitudinal muscle contracts simultaneously, as suggested by Stevens et al, or whether the neural network ensures that it does not contract while the circular muscle is contracting. If the longitudinal muscle did indeed contract synchronously with the circular, it would violate biological principles of energy conservation because contraction would be against the opposing forces of circular muscle contraction. Conversely, simultaneous contraction of longitudinal and circular muscle in the propulsive segment would be expected to increase wall tension additively and this may have some adaptive advantage; nevertheless, it is unclear what this might be.

J D WOOD

Professor,

Departments of Physiology and Internal Medicine, The Ohio State University College of Medicine, 302 Hamilton Hall, 1645 Neil Avenue, Columbus, Ohio 43210, USA email:wood.13@osu.edu

1 Kottegoda SR. An analysis of possible nervous mechanisms involved in the peristaltic reflex. F Physiol Lond 1969;200:525-41.

2 Gregory JE, Bentley JE. The peristaltic reflex in the isolated guinea-pig ileum during drug induced spasm of the longitudinal muscle. Aust $\mathcal{F}$ Exp Biol Med Sci 1968;46:1-16.

3 Wood JD, Perkins WE. Mechanical interactions between longitudinal and circular axes of the small intestine. Am F Physiol 1970;218:762-8.

4 McGroarty FC, McKirdy HC. Preliminary observations on the functional relationship of the longitudinal and circular laver of the muscularis externa of the rabbit rectum using a flat preparation. F Physiol Lond 1971;214:19P.

5 Raiford TS, Mulinos MG. Studies in gastrointestinal motility. Arch Surg 1936;33:276-96.

6 Wood JD. Gastrointestinal motility. In: Rhoades RA, Tanner GA, eds. Medical physiology. Boston: Little, Brown and Co, 1995:505-29.

7 Wood JD. Physiology of the enteric nervous system. In: Johnson LR, Alpers DH, Christensen J, Jacobson Walsh JH, eds. Physiology of the gastrointestinal tract. New York: Raven Press, 1994:423-82.

tract. New York: Raven Press, 1994:423-82.
Kosterlitz HW. Intrinsic intestinal reflexes. Am 7 Dig Dis 1967;12:245-54

8 Kosterlitz HW. Intrinsic intestinal reflexes. Am f Dig Dis 1967;12:245-54. Brann L, Wood JD. Motility of
J Dig Dis 1976; 21:633-40.

10 Pouderoux P, Lin SZ, Kahrilas PJ. Timing, propagation, coordination, and effect of esophageal shortening during peristalsis. Gastroenterology 1997; 112: $1147-54$. 